\title{
Effect of Genetic Modifications on Physical and Functional Titers of Adenoviral Cancer Gene Therapy Constructs
}

\section{Heiniö, Camilla}

2019-06-01

Heiniö , C , Sorsa , S , Siurala , M , Grönberg-Vähä-Koskela, S, Havunen , R , Haavisto , E , Koski , A , Hemminki , O, Zafar, S, Cervera-Carrascon, V , Munaro, E , Kanerva , A \& Hemminki , A 2019 , ' Effect of Genetic Modifications on Physical and Functional Titers of Adenoviral Cancer Gene Therapy Constructs ' , Human Gene Therapy , vol. 30 , no. 6 , pp. 740-752 . https://doi.org/10.1089/hum.2018.240

http://hdl.handle.net/10138/309908

https://doi.org/10.1089/hum.2018.240

unspecified

acceptedVersion

Downloaded from Helda, University of Helsinki institutional repository.

This is an electronic reprint of the original article.

This reprint may differ from the original in pagination and typographic detail.

Please cite the original version. 


\section{Effect of genetic modifications on physical and functional titers of adenoviral cancer gene therapy constructs}

Camilla Heiniö ${ }^{1}$, Suvi Sorsa ${ }^{1,2}$, Mikko Siurala ${ }^{1,2}$, Susanna Grönberg-Vähä-Koskela ${ }^{1}$, Riikka Havunen $^{1,2}$, Elina Haavisto ${ }^{2}$, Anniina Koski-Palkén ${ }^{1}$, Otto Hemminki ${ }^{1,3}$, Sadia Zafar ${ }^{1}$, Víctor Cervera-Carrascon $^{1,2}$, Eleonora Munaro ${ }^{1}$, Anna Kanerva ${ }^{1,4}$ and Akseli Hemminki ${ }^{1,2,5,}$

${ }^{1}$ Cancer Gene Therapy Group, Faculty of Medicine, University of Helsinki, Helsinki, Finland

${ }^{2}$ TILT Biotherapeutics Ltd, Helsinki, Finland

${ }^{3}$ Helsinki University Hospital, Department of Urology

${ }^{4}$ Department of Obstetrics and Gynecology Helsinki University Hospital

${ }^{5}$ Helsinki University Hospital Comprehensive Cancer Center

Correspondence should be addressed to A.H. (akseli.hemminki@helsinki.fi)

Haartmaninkatu 3, 00290 Helsinki, Finland

Phone: +358 0294125464

Keywords: adenovirus, vector, gene therapy, cancer, oncolytic virus 


\begin{abstract}
After discovery and characterization of the adenovirus in the 1950 s, this prevalent cause of the common cold and other usually mild diseases has been modified and utilized in biomedicine in several ways. To date, adenoviruses are the most frequently used vectors and therapeutic (e.g. oncolytic) agents with a number of beneficial features. They infect both dividing and non-dividing cells, enable high-level, transient protein expression, and are easy to amplify to high concentrations. As an important and versatile research tool, it is of essence to understand the limits and advantages that genetic modification of adenovirus vectors may entail. Therefore, we performed a retrospective analysis of adenoviral gene therapy constructs produced in the same laboratory with similar methods. The aim was to assess the impact of various modifications on the physical and functional titer of the virus.
\end{abstract}

We found that, genome size (designed within "the 105\% golden rule"), did not significantly affect the physical titer of the adenovirus preparations, regardless of the type of transgene (e.g. immunostimulatory vs other), number of engineered changes, and size of the mutated virus genome. One statistically significant exception was noted, however. Chimeric adenoviruses (5/3) had a slightly lower physical titer compared to Ad5-based viruses, although a trend for the opposite was true for functional titer. Thus, $5 / 3$ chimeric viruses may in fact be appealing from the safety versus efficacy viewpoint. Armed viruses had lower, both functional and physical, titers than unarmed viruses, while five genomic modifications started to decrease functional titer. Importantly, even highly modified armed viruses generally had good titers compatible with clinical testing.

In summary, this paper shows the plasticity of adenovirus for various vector, oncolytic, and armed oncolytic uses. These results inform future generations of adenovirus-based drugs for human use. This information is directly transferable to academic laboratories and the biomedical industry involved in vector design and production optimization. 


\section{Introduction}

Genetic engineering methods have enabled the modification of wild-type viruses for use as safe and effective gene therapy vectors for multiple diseases ${ }^{1}$. Among the most thoroughly-characterized gene therapy vectors is the adenovirus, which also continues to be the most popular ${ }^{2}$. Wild-type adenoviruses commonly infect the upper respiratory, gastrointestinal and urinary tracts, or the conjunctiva, causing mild and self-limiting disease. Occasionally, pneumonia or even encephalitis can occur in immunosuppressed individuals or children. Of note, rare cases of systemic dissemination of adenovirus demonstrate the ability of the virus to travel through blood into distant tissues, which has implications for therapeutic use of tumor-selective viruses ${ }^{3}$. In this regard, it is not surprising that transduction of non-injected metastases has been seen in cancer patients ${ }^{3-5}$.

Adenovirus is a non-enveloped double-stranded DNA virus with a protein capsid ${ }^{6}$. The capsid structure encloses a genome consisting of roughly 36 kilobase $(\mathrm{kb})$ pairs ${ }^{7}$. Conveniently, the virion structure allows insertion of complementary DNA (cDNA) elements for expression of foreign transgenes. In addition to immunostimulatory transgenes (e.g. cytokines and chemokines), nonimmunostimulatory transgenes (e.g. tagging proteins such as luciferase, GFP, or prodrug converting enzymes, and ion channels), and cDNA coding for full-length antibodies (e.g. anti-CTLA4 and antiHER2) can be inserted into the adenovirus genome to generate recombinant adenoviral vectors ${ }^{8,9}$.

For therapeutic gene transfer purposes, replication-deficient adenovirus vectors can be engineered. In this approach, adenoviral early genes (E1-E4, often just E1 and E3) are deleted and replaced with a transgene of interest ${ }^{6}$. The inserted genes are typically placed under a constitutive promoter element, such as the cytomegalovirus (CMV) or Rous sarcoma virus (RSV) promoter, which allow transgene expression in any transduced cell. For example, Advexin is an E1/E3-deleted serotype 5 adenovirus expressing wild-type $\mathrm{p} 53$ under $\mathrm{CMV}^{10}$. A similar virus, Gendicine, has been approved for routine use in China. 
In another embodiment of the vector concept, adenoviruses coding for immunostimulatory cytokines have been constructed and used clinically for various cancer types; AdCAIL-2 and TG1024 are both interleukin-2 (IL-2) -encoding non-replicating vectors explored in the treatment of melanoma ${ }^{11,12}$. Also several other cytokines have been inserted, including tumor necrosis factor alpha $(\mathrm{TNFa})^{13}$, interferon beta (IFNb) ${ }^{14}$, and interferon gamma (IFNg) ${ }^{15}$. Prodrug converting enzymes have also been a popular approach, such as $\mathrm{rAd} / \mathrm{CPG} 2(\mathrm{Q} 3)$-Thy1 coding for GPI anchored, prodrug converting carboxypeptidase G2 ${ }^{16}$. Several vector approaches have been promising enough to make it to randomized phase 3 testing, but adenoviral virotherapy agents for cancer treatment have not been approved outside of China yet ${ }^{13}$.

In contrast to replication-deficient viruses, replication-competent oncolytic viruses are able to go through their entire life cycle in a tumor but not in normal cells, culminating in cell lysis and release of new virions. Tumor specificity can be achieved on the level of replication with tumor-specific promoters or deletions transcomplemented in tumor cells (transcriptional targeting). In addition, the virus capsid can be modified to increase infection of tumor cells, or to overcome lack of receptors that are down-regulated in advanced cancers (transductional targeting) ${ }^{6,17,18}$.

In the former approach, tumor tissue -active promoters, such as human telomerase reverse transcriptase (hTERT), carcinoembryonic antigen (CEA), cyclooxygenase (Cox-2), and E2F, have been used in oncolytic adenovirus constructs ${ }^{19-22}$. Deletions of E1b55k or E1A constant region 2 have been employed to restrict the virus replication to cells defective in the $\mathrm{p} 14 \mathrm{ARF} / \mathrm{p} 53$ or $\mathrm{p} 16 / \mathrm{Rb}$ pathways, respectively. Oncorine, an oncolytic adenovirus approved for the treatment of head and neck cancer in China, is an example of the former type of deletion mutant. Of note, it is logical to combine the benefits of vectored transgene delivery with the oncolytic platform capable of local amplification and systemic dissemination to metastases ${ }^{4}$. 
In addition to transcriptional targeting, the adenovirus fiber protein can be modified to feature either synthetic elements such as RGD-4C or structures from different serotypes. Often the goal is to avoid reliance on the Ad5 receptor (coxsackie adenovirus receptor CAR), which is known to be almost universally down-regulated in advanced metastatic tumors due to its role in cellular adhesion ${ }^{23}$. To solve this issue, several adenovirus variations have been explored, such as $\mathrm{Ad} 5 / 7^{24}, \mathrm{Ad} 5 / 11^{25}$, Ad5/35 ${ }^{26}$ and Ad3/Ad11p ${ }^{27}$. The clinically most studied chimera, however, is the $5 / 3$ structure which features the knob of serotype 3 in an otherwise Ad5 virion ${ }^{22,23,28,29}$. Again, there is no reason to avoid combining the benefits of transductional targeting with the armed oncolytic platform ${ }^{30}$.

Heretofore, a systematic analysis of the effects of adenovirus modifications (i.e. capsid structure, transgene type, and overall number of changes) on viral vector yield has not been performed. We gathered physical and functional titer data of circa 50 different adenoviral gene therapy constructs produced in the Cancer Gene Therapy Group (CGTG) at the University of Helsinki between 2002 and 2018 to assess the impact of various modifications on virus titers. Additionally, since it has been suggested that the amount of DNA that can be packaged into adenovirus virions is limited ${ }^{31,32}$, we performed correlation analysis to evaluate the effect of genome size on virus titers. 


\section{Materials and Methods}

\section{Adenoviruses}

Adenovirus construction and production is described in detail in the sources found in Table $\mathbf{1 .}$ Typically, non-replicative viruses were constructed by insertion of a transgene into a cloning plasmid, which was then co-transfected with a "rescue plasmid" (containing the rest of the virus genome) into bacteria featuring the requisite eukaryotic recombination enzymes. Linearized plasmid was then propagated in e.g. HEK293 cells, which provide the E1 region in trans ${ }^{23}$. Many replication competent (oncolytic) chimeric adenoviruses were constructed by an adapted BAC- recombineering method ${ }^{33-}$ ${ }^{35}$, and propagated in non-transcomplementing A549 cells to avoid the risk of back-recombination generating a modified but non-tumor selective virus. Produced virions were collected from cells showing cytopathic effect by trypsin detaching or scraping, centrifugation, and repeated freeze-thaw cycles. Downstream purification employed two consecutive cesium chloride density centrifugations followed by dialysis to remove cesium chloride.

Physical titer (virus particles, VP) was assessed spectrophotometrically (OD260 reading). Functional titering was done with Tissue Culture Infectious Dose (TCID50) assay in HEK293 or A549 cells, depending on virus type. TCID50 titers were converted into a more conventional plaque forming units (PFU) titer using a formula ${ }^{18,36}$. VP/PFU ratios (physical to functional titer) were calculated by dividing VP titer with PFU titer.

\section{Statistical analyses}

All virus constructs included in statistical analyses are listed in Table 1. All statistical analyses, Kruskal-Wallis and Spearman correlation were performed using GraphPad Prism 7 (GraphPad Software Inc., San Diego, CA, USA). 


\section{Results}

Chimeric 5/3 adenoviruses have moderately lower physical, but not functional, titers compared with fully serotype 5 viruses

Viruses with fully serotype 5 capsid structure had the highest VP titers with an arithmetic mean of $4.2 \times 10^{12} \mathrm{VP} / \mathrm{ml}$ and median of $2.3 \times 10^{12} \mathrm{VP} / \mathrm{ml}(\mathrm{n}=30$ virus preparations) (Figure 1a, Figure 1c). Chimeric $5 / 3$ viruses had lower titers with a mean of $2.4 \times 10^{12} \mathrm{VP} / \mathrm{ml}$ and median of $1.1 \times 10^{12}(\mathrm{n}=$ 88 ), and the difference to serotype 5 viruses was statistically significant ( $p=0.0412$, KruskalWallis).Viruses with other capsid modifications (RGD, pk7, pk21, RGD + pk7) had a similar trend for moderately lower VP titers (mean value $2.4 \times 10^{12} \mathrm{VP} / \mathrm{ml}$, median $=1,9 \times 10^{12}, \mathrm{n}=6$, not statistically significant; note smaller n) versus fully Ad5.

Functional titers were not significantly different between fully Ad5, chimeric 5/3, and other capsidmodified adenoviruses. Ad5 mean PFU titer was $1.1 \times 10^{11} \mathrm{PFU} / \mathrm{ml}, 5 / 3$ chimeric virus mean was more than twice as high $\left(2.5 \times 10^{11} \mathrm{PFU} / \mathrm{ml}\right)$, while the mean of the other capsid modification group was lower $\left(4.4 \times 10^{10} \mathrm{PFU} / \mathrm{ml}\right)$. The respective medians were: serotype $5=9.0 \times 10^{10} \mathrm{PFU} / \mathrm{ml}$, chimeric 5/3 $=4.0 \times 10^{10} \mathrm{PFU} / \mathrm{ml}$, other modifications $\left.=4.8 \times 10^{10} \mathrm{PFU} / \mathrm{ml}\right)($ Figure 1b, Figure 1d). Ratios of physical to functional titer were not significantly different between groups (Figure 1e). The ratio was 37.6 for Ad5 and 35.2 for Ad5/3. Because some clear outliers were identified, medians were used for ratio calculations. Regarding outliers, Ad5/3lucS ${ }^{37}$ with shaft mutations, had ratios ranging from 11483 to 79556 , suggesting that this capsid configuration (or other properties of the virion) did interfere with virus production and function. (Figure 1e).

\section{Transgene type significantly affects functional virus titers}


Comparison of physical VP titers of viruses with different transgene types did not reveal significant differences (Kruskal-Wallis) (Figure 2a). It is noteworthy, however, that the functional titer of virus groups with immunostimulatory $(p=0.0054)$, non-immunostimulatory transgenes $(p=0.0403)$ and antibody ( $\mathrm{p}=0.0131)$ transgenes were significantly lower compared with the control group of viruses with no added transgenes (Figure 2b). Comparison of transgene groups revealed significantly lower ratios for the immunostimulatory group $(p=0.0016)$ and antibody groups $(p=0.027)$ compared with the group with no transgenes (Figure 2c).

\section{Number of modifications affects virus functionality}

Viruses were grouped according to the number of functionally relevant, engineered, modifications. Kruskall-Wallis analysis did not indicate statistically significant differences between the number of modifications and physical/functional titers, or the ratios between physical and functional titers when fewer than 5 modifications were done. However, five genetic modifications caused a significant drop in the physical and functional titers $(\mathrm{p}=0.139$ and $\mathrm{p}=0.002$ respectively) (Figure 3 and

\section{Supplementary Table 1).}

\section{Genome size does not correlate with physical or functional virus titer}

We performed Spearman correlation analysis comparing functional titers with genome sizes. Wildtype adenovirus serotype 5 (Ad300wt strain purchased from ATCC) served as $100 \%$ reference value. When genome size was plotted against physical titer, we observed no correlation between the two factors $(r=0.04)$ (Figure 4a). Similar results, indicating a lack of correlation, were observed with functional titer $(r=-0.06)$ (Figure 4b) and VP/PFU ratio $(r=-0.03)$ (Figure 4c). Of note, we have 
not attempted to make viruses whose genome size would be smaller than $98 \%$ or larger than $105 \%$ of the wild-type genome, and it seems unwise to extrapolate our findings beyond these limits. 


\section{Discussion}

The construction and production of biological therapeutic agents for human use is a heavily regulated field with a high demand of accuracy in order to provide safety and reliable drugs. Importantly, as adenoviruses are being utilized for several clinical and preclinical purposes, it is of essence to understand the effects of man-made modifications to the packaging ability of the virus. One of the main reasons for conducting modifications to adenovirus vectors relates to the concern regarding preexisting immunity against the vector and subsequent vector-depleting immune response and toxicity $^{38}$.

The adenovirus capsid consists mainly of three components (fiber, penton and hexon proteins), which are available for neutralizing antibodies. Of these, the fiber proteins alone do not seem to function as major targets for neutralizing antibodies, since the hexon proteins have been shown to be important immunoneutralizing targets as well ${ }^{39}$. Therefore, it is expected that while chimerism and other modifications to the fiber allow a degree of escape from pre-existing antibodies ${ }^{40}$, they do not dramatically affect the immunogenicity of the viruses. Thus, over time antibodies will develop also against modified capsids. Of note, with increased understanding of selecting the right vector for each purpose, adenovirus has been very safe in the context of cancer therapy ${ }^{39,41-43}$. Immunogenicity is an important tool when inducing anti-tumor immunity while it might be a caveat when treating hereditary disease, for example.

In cancer therapy, the antiviral immune response is harnessed as a positive, immune system activating, tumor depleting force in the battle against difficult-to-treat tumors. In this regard, some early studies identified key issues regarding vector design, such as the association of genome size and virion stability ${ }^{7,31,32}$. They concluded that adenovirus vectors are unstable at sizes over $39 \mathrm{~kb}$, which is roughly $105 \%$ of the wild-type adenovirus genome size. This result has subsequently been applied as a golden standard when adenovirus vectors have been developed and designed. 
Interestingly, however, even though many types of oncolytic and vector adenoviruses have been constructed, it is not known how modification type, the number of engineered modifications, capsid type, or replication competence affect stability and packaging of the virus. Lack of understanding of these issues is a major caveat in the field, since on one hand, it is easy to attempt to construct almost any type of virus, but on the other, many of the modifications that can be envisioned, might easily affect virus assembly.

For example, capsid is one of the key determinants in virion stability, so any changes to it could reduce titers. Moreover, transgenes added to the virus genome could potentially affect producer cells. Subsequently, it would not be surprising if the number of modification would eventually decrease the ability of the virus to replicate. In the course of evolution, each of the 57 adenovirus serotypes discovered to date, have been optimized for stability and replication. Thus, man made, engineered changes could result in decrease in these attributes. The adenovirus genome is read in both directions, and most of the DNA is coding sequence, indicating that any change can have unexpected changes in other adenovirus proteins.

Therefore, we analyzed the effect of modifications in vectors used and/or made in CGTG at the University of Helsinki. Several modifications to the adenovirus fiber and/or knob have been employed, altering the specificity and efficacy of infection. In this study, we found that virions with chimeric 5/3 fiber modifications produced a slightly but statistically significantly lower physical titer compared with virus preparations produced with unmodified Ad5 knob and shaft domains. In contrast, mean functional titers seemed higher with chimeric viruses. This is in accord with previous findings indicating that chimeric viruses result in a higher rate of oncolysis, virus DNA to nucleus transportation, and more efficient replication efficacy compared with control viruses ${ }^{44}$.

Efficacy, however, is a multifactorial endpoint influenced not only by virus replication and lysis of cells, but also infection dynamics related to expression of the relevant receptors. There is much data 
indicating that the receptors for Ad5/3, such as desmoglein 2, are expressed to higher degree on tumor cells than the Ad5 receptor CAR ${ }^{17,44,45}$. Additionally, some data indicate that $5 / 3$ chimerism also enhances replication efficiency ${ }^{44}$, thus other than chimeric modifications made to the analyzed virus preparations seem to hamper the previously noted enhanced virion production.

Importantly, a lower physical to functional particle ratio may be advantageous for human use. Adverse events are known to be associated with physical titer, while efficacy is associated with functional titer. For these reasons, physical titering is recommended in clinical trials as safety is even more important than efficacy ${ }^{46}$.

Of note, the titers reported here are not the result of optimization of production. Instead, in most academic laboratories, students, technicians, and other researchers produce viruses themselves, with limited optimization of the process. At CGTG, we do perform a panel of quality control assays on each virus batch produced, and batches not fulfilling these criteria are discarded. However, process development has not been the most important issue in preclinical stages and academic processes, since standard methods generally give good adenovirus yields. In contrast, when moving on to clinical trials, it is important to minimize the proportion of inactive particles (which influence adverse events but not efficacy), emphasizing the importance of process development in virus production. Testing the multiplicity of infection, cellular confluency, the cell line used, infection medium, harvest time, and other details can increase yield dramatically ${ }^{47}$.

The observed production efficacy was similar when the group of "other capsid modifications" was compared with wild-type-capsid virion preparations. The other capsid modifications group contains several different knob protein modified virions, thus, these modifications might have altered the stability of the virions in the production system. Although fiber protein modifications of Ad2 have been shown to impact the assembly of the virion ${ }^{48}$, a negative effect of knob protein modifications on virion stability could be less obvious, because the knob proteins are located on the outermost parts 
of the virion. Additionally, several studies have noted a higher infectivity of knob modified Ad5, which would not have been seen if the knob modified virions were very unstable ${ }^{17,49}$.

We hypothesized that when transgenes are inserted into the adenovirus genome, titers could be influenced. Transgene products are produced during virus replication, which might reduce the ability of the cell to produce viral proteins, assuming an overall limited production capacity. Alternatively, the transgene product itself might affect the host's signaling cascades, eventually either hampering or - less likely - enhancing the virion production efficacy. Therefore, the transgenes in this study were grouped and analyzed with regard to virus titers. We found that the type of insert did not impact the number of produced total virions. However, the production of functional (PFU) virions was significantly lowered (e.g. immunostimulatory vs no transgene). Therefore, this indicates that transgenes can affect the host cell production thus impeding optimal production and/or folding of virion peptides, the correct assembly of the virion, or some other yet unidentified part of proper virus production.

The effect of the number of modifications was examined in detail. No significant differences in physical- and functional titers between preparations of viruses with zero to four modifications was observed. However, the titer was significantly lowered when five modifications were made to virus genomes. This might be caused by a variety of reasons. For example, several signaling sequences are imbedded into the genome, and the sequence in itself can function as a stabilizing entity ${ }^{50}$. Alternatively or in addition, some other yet unknown functions of the sequence might have been disturbed unknowingly while modifying the genome.

As previous studies by Kennedy and Parks had shown that genome size exceeding $105 \%$ is undesirable ${ }^{7}$, all virus preparations made in this study were constructed within this rule. Additionally, it has been suggested that smaller genome size correlates with poorer heat stability of adenovirus virions and if crucial virion proteins are deleted, helper viruses are needed. Thus, this indicates that 
deleting too much can be counterproductive. However, in the study conducted by Kennedy and Parks, the virions also contained additional modifications, which might have affected results ${ }^{7}$. However, it is maybe not so interesting to make smaller viruses, because often the aim is to use the virus to deliver a payload, which requires adding something to the virus.

Thus, it was of interest to see how sensitive virion production was to size changes within the $105 \%$ "golden rule". This analysis revealed that size of the studied virus genomes did not affect the physical or functional virus titers. When adenoviruses are assembled, the electrostatic interactions between DNA and the capsid proteins are neutralized by the basic adenoviral proteins VII, V, and $\mu$, thus probably contributing to the noted flexibility of the adenovirus virions ${ }^{51}$.

With replication-competent viruses, the input titer is just one variable. With each tumor cell producing tens of thousands of new virions, titer may not be the critical determinant of overall efficacy. Instead, when designing viruses for cancer treatment, cellular, intratumoral, and anti-viral issues may be more relevant. Also, intratumoral complexities such as extracellular matrix, hyperbaric, necrotic, hypoxic, and acidic areas may thwart spreading of the virus ${ }^{52}$. Of note, the production of big proteins such as antibodies and immunostimulatory proteins, seems to be a burden to functional virion production, and thus needs the attention from virologists in order to solve this issue. In conclusion, the data collected in this study reveals that adenovirus can be modified quite flexibly without clinically relevant loss of functional titer. 


\section{Acknowledgements}

This study was supported by Jane and Aatos Erkko foundation, HUCH Research Funds (EVO), Sigrid Juselius Foundation, Finnish Cancer Organizations, University of Helsinki and TILT Biotherapeutics Ltd. The University of Helsinki Biostatistics consultation unit (Paula Bergman) is acknowledged for their support in biostatistical conundrums. 


\section{References}

1. Appaiahgari MB, Vrati S. Adenoviruses as gene/vaccine delivery vectors: promises and pitfalls. Expert Opinion on Biological Therapy. 2015;15(3):337-351.

2. Ginn SL, Amaya AK, Alexander IE, Edelstein M, Abedi MR. Gene therapy clinical trials worldwide to 2017: An update. J Gene Med. 2018;20(5):e3015.

3. Garcia M, Moreno R, Gil M, et al. A phase I trial of oncolytic adenovirus ICOVIR-5 administered intravenously to cutaneous and uveal melanoma patients. Hum Gene Ther. 2018.

4. Koski A, Bramante S, Kipar A, et al. Biodistribution Analysis of Oncolytic Adenoviruses in Patient Autopsy Samples Reveals Vascular Transduction of Noninjected Tumors and Tissues. Mol Ther. 2015;23(10):1641-1652.

5. Garcia-Carbonero R, Salazar R, Duran I, et al. Phase 1 study of intravenous administration of the chimeric adenovirus enadenotucirev in patients undergoing primary tumor resection. J Immunother Cancer. 2017;5(1):71.

6. Kanerva A, Hemminki A. Modified adenoviruses for cancer gene therapy. Int $\mathrm{J}$ Cancer. 2004;110(4):475-480.

7. Kennedy MA, Parks RJ. Adenovirus virion stability and the viral genome: size matters. Mol Ther. 2009;17(10):1664-1666.

8. Liikanen I, Tahtinen S, Guse K, et al. Oncolytic Adenovirus Expressing Monoclonal Antibody Trastuzumab for Treatment of HER2-Positive Cancer. Mol Cancer Ther. 2016;15(9):2259-2269.

9. Dias JD, Hemminki O, Diaconu I, et al. Targeted cancer immunotherapy with oncolytic adenovirus coding for a fully human monoclonal antibody specific for CTLA-4. Gene Ther. 2012;19(10):988998.

10. Chen GX, Zhang S, He XH, Liu SY, Ma C, Zou XP. Clinical utility of recombinant adenoviral human p53 gene therapy: current perspectives. Onco Targets Ther. 2014;7:1901-1909.

11. Dummer R, Rochlitz C, Velu $\mathrm{T}$, et al. Intralesional adenovirus-mediated interleukin-2 gene transfer for advanced solid cancers and melanoma. Mol Ther. 2008;16(5):985-994.

12. Stewart AK, Lassam NJ, Quirt IC, et al. Adenovector-mediated gene delivery of interleukin-2 in metastatic breast cancer and melanoma: results of a phase 1 clinical trial. Gene Ther. 1999;6(3):350363.

13. Kali A. TNFerade, an innovative cancer immunotherapeutic. Indian J Pharmacol. 2015;47(5):479-483.

14. Sterman DH, Recio A, Haas AR, et al. A phase I trial of repeated intrapleural adenoviral-mediated interferon-beta gene transfer for mesothelioma and metastatic pleural effusions. Mol Ther. 2010;18(4):852-860.

15. Dreno B, Urosevic-Maiwald M, Kim Y, et al. TG1042 (Adenovirus-interferon-gamma) in primary cutaneous B-cell lymphomas: a phase II clinical trial. PLoS One. 2014;9(2):e83670.

16. Cowen RL, Williams JC, Emery S, et al. Adenovirus vector-mediated delivery of the prodrugconverting enzyme carboxypeptidase G2 in a secreted or GPI-anchored form: High-level expression of this active conditional cytotoxic enzyme at the plasma membrane. Cancer Gene Ther. 2002;9(11):897-907.

17. Kanerva A, Hemminki A. Adenoviruses for treatment of cancer. Ann Med. 2005;37(1):33-43.

18. Havunen R, Siurala M, Sorsa S, et al. Oncolytic Adenoviruses Armed with Tumor Necrosis Factor Alpha and Interleukin-2 Enable Successful Adoptive Cell Therapy. Molecular Therapy - Oncolytics. 2017;4:77-86.

19. Diaconu I, Cerullo V, Hirvinen ML, et al. Immune response is an important aspect of the antitumor effect produced by a CD40L-encoding oncolytic adenovirus. Cancer Res. 2012;72(9):2327-2338.

20. Li Y, Chen Y, Dilley J, et al. Carcinoembryonic antigen-producing cell-specific oncolytic adenovirus, OV798, for colorectal cancer therapy. Mol Cancer Ther. 2003;2(10):1003-1009.

21. Pesonen $S$, Nokisalmi $P$, Escutenaire $S$, et al. Prolonged systemic circulation of chimeric oncolytic adenovirus Ad5/3-Cox2L-D24 in patients with metastatic and refractory solid tumors. Gene Ther. 2010;17(7):892-904.

22. Hemminki O, Parviainen S, Juhila J, et al. Immunological data from cancer patients treated with Ad5/3-E2F-Delta24-GMCSF suggests utility for tumor immunotherapy. Oncotarget. 2015;6(6):44674481 . 
23. Kanerva A, Wang M, Bauerschmitz GJ, et al. Gene transfer to ovarian cancer versus normal tissues with fiber-modified adenoviruses. Mol Ther. 2002;5(6):695-704.

24. Gall J, Kass-Eisler A, Leinwand L, Falck-Pedersen E. Adenovirus type 5 and 7 capsid chimera: fiber replacement alters receptor tropism without affecting primary immune neutralization epitopes. J Virol. 1996;70(4):2116-2123.

25. Wei LM, Cao S, Yu WD, Liu YL, Wang JT. Overexpression of CX3CR1 is associated with cellular metastasis, proliferation and survival in gastric cancer. Oncol Rep. 2015;33(2):615-624.

26. Liu Y, Wang H, Yumul R, et al. Transduction of liver metastases after intravenous injection of Ad5/35 or Ad35 vectors with and without factor X-binding protein pretreatment. Hum Gene Ther. 2009;20(6):621-629.

27. Kuhn I, Harden P, Bauzon M, et al. Directed evolution generates a novel oncolytic virus for the treatment of colon cancer. PLoS One. 2008;3(6):e2409.

28. Kim KH, Dmitriev IP, Saddekni S, et al. A phase I clinical trial of Ad5/3-Delta24, a novel serotypechimeric, infectivity-enhanced, conditionally-replicative adenovirus (CRAd), in patients with recurrent ovarian cancer. Gynecol Oncol. 2013;130(3):518-524.

29. Kuryk L, Haavisto E, Garofalo M, et al. Synergistic anti-tumor efficacy of immunogenic adenovirus ONCOS-102 (Ad5/3-D24-GM-CSF) and standard of care chemotherapy in preclinical mesothelioma model. Int J Cancer. 2016;139(8):1883-1893.

30. Bauerschmitz GJ, Guse K, Kanerva A, et al. Triple-targeted oncolytic adenoviruses featuring the cox2 promoter, E1A transcomplementation, and serotype chimerism for enhanced selectivity for ovarian cancer cells. Mol Ther. 2006;14(2):164-174.

31. Ghosh-Choudhury G, Haj-Ahmad Y, Graham FL. Protein IX, a minor component of the human adenovirus capsid, is essential for the packaging of full length genomes. Embo j. 1987;6(6):17331739.

32. Bett AJ, Prevec L, Graham FL. Packaging capacity and stability of human adenovirus type 5 vectors. J Virol. 1993;67(10):5911-5921.

33. Warming S, Costantino N, Court DL, Jenkins NA, Copeland NG. Simple and highly efficient BAC recombineering using galK selection. Nucleic Acids Res. 2005;33(4):e36.

34. Ruzsics Z, Wagner M, Osterlehner A, Cook J, Koszinowski U, Burgert HG. Transposon-assisted cloning and traceless mutagenesis of adenoviruses: Development of a novel vector based on species D. J Virol. 2006;80(16):8100-8113.

35. Muck-Hausl M, Solanki M, Zhang W, Ruzsics Z, Ehrhardt A. Ad 2.0: a novel recombineering platform for high-throughput generation of tailored adenoviruses. Nucleic Acids Res. 2015;43(8):e50.

36. Wulff NH, Tzatzaris M, Young PJ. Monte Carlo simulation of the Spearman-Kaerber TCID50. J Clin Bioinforma. 2012;2(1):5.

37. Koski A, Karli E, Kipar A, Escutenaire S, Kanerva A, Hemminki A. Mutation of the fiber shaft heparan sulphate binding site of a 5/3 chimeric adenovirus reduces liver tropism. PLoS One. 2013;8(4):e60032.

38. Raper SE, Yudkoff M, Chirmule N, et al. A pilot study of in vivo liver-directed gene transfer with an adenoviral vector in partial ornithine transcarbamylase deficiency. Hum Gene Ther. 2002;13(1):163175.

39. Schagen FH, Ossevoort M, Toes RE, Hoeben RC. Immune responses against adenoviral vectors and their transgene products: a review of strategies for evasion. Crit Rev Oncol Hematol. 2004;50(1):5170.

40. Sarkioja M, Pesonen S, Raki M, et al. Changing the adenovirus fiber for retaining gene delivery efficacy in the presence of neutralizing antibodies. Gene Ther. 2008;15(12):921-929.

41. Pesonen S, Diaconu I, Cerullo V, et al. Integrin targeted oncolytic adenoviruses Ad5-D24-RGD and Ad5-RGD-D24-GMCSF for treatment of patients with advanced chemotherapy refractory solid tumors. 2012;130(8):1937-1947.

42. Cerullo V, Pesonen S, Diaconu I, et al. Oncolytic adenovirus coding for granulocyte macrophage colony-stimulating factor induces antitumoral immunity in cancer patients. Cancer Res. 2010;70(11):4297-4309.

43. Kanerva A, Koski A, Liikanen I, et al. Oncolytic adenovirus coding for granulocyte macrophage colony-stimulating factor induces antitumoral immunity in cancer patients. Cancer Research. 2015;70(11). 
44. Kawakami Y, Li H, Lam JT, Krasnykh V, Curiel DT, Blackwell JL. Substitution of the adenovirus serotype 5 knob with a serotype 3 knob enhances multiple steps in virus replication. Cancer Res. 2003;63(6):1262-1269.

45. Wang H, Li ZY, Liu Y, et al. Desmoglein 2 is a receptor for adenovirus serotypes 3, 7, 11 and 14. Nat Med. 2011;17(1):96-104.

46. EMEA, European, Medicine, Agency. ICH Considerations Oncolytic Viruses. 2009; http://www.emea.europa.eu.

47. Kamen A, Henry O. Development and optimization of an adenovirus production process. J Gene Med. 2004;6 Suppl 1:S184-192.

48. Levine AJ, Ginsberg HS. Mechanism by which fiber antigen inhibits multiplication of type 5 adenovirus. J Virol. 1967;1(4):747-757.

49. Wu H, Seki T, Dmitriev I, et al. Double Modification of Adenovirus Fiber with RGD and Polylysine Motifs Improves Coxsackievirus-Adenovirus Receptor-Independent Gene Transfer Efficiency. 2002;13(13):1647-1653.

50. Gräble $M$, Hearing $P$. Adenovirus type 5 packaging domain is composed of a repeated element that is functionally redundant. J Virol. 1990;64(5):2047-2056.

51. Saha B, Wong CM, Parks RJ. The adenovirus genome contributes to the structural stability of the virion. Viruses. 2014;6(9):3563-3583.

52. Berkey SE, Thorne SH, Bartlett DL. Oncolytic Virotherapy and the Tumor Microenvironment. Adv Exp Med Biol. 2017;1036:157-172. 